\title{
Devious signals from NFKB driving breast cancer progression
}

\author{
Christopher C. Benz $z^{a, b}$ \\ ${ }^{a}$ Buck Institute for Age Research, Novato; ${ }^{b}$ Department of Medicine and Comprehensive Cancer Center, \\ University of California, San Francisco, CA, USA.
}

\section{Citation of original article 1:}

P. Bhatia, M. M. Sanders, M. F. Hansen. Expression of receptor activator of nuclear factor-kappaB is inversely correlated with metastatic phenotype in breast carcinoma. Clinical Cancer Research 2005; 11(1): 162-5.

\begin{abstract}
of the original article 1
During normal bone remodeling, the receptor activator of nuclear factor-kappaB (RANK) interacts with its ligand RANKL, which is present on pre-osteoclasts, resulting in bone resorption and initiation of new bone formation. When breast cancer metastasizes to bone, normal bone remodeling is disturbed by invasion of tumor cells, resulting in osteolytic lesions. We have studied the expression of both RANK and RANKL in 10 nonneoplastic breast samples, 58 infiltrating ductal carcinoma (IDC), and 43 breast cancer bony metastases (BTM). RANK seemed to be present in all samples tested. However, whereas RANKL expression was observed in $90 \%$ of nonneoplastic breast, RANKL expression was only observed in $62 \%$ of nonmetastatic IDC, $31 \%$ of metastatic IDC, and $2 \%$ of osteolytic BTM lesions. This decreased or absent expression of RANKL in the tumor cells may allow RANK, which is normally expressed as a receptor on the cell surface, to target RANKL present on the cell surface of normal osteoblasts and stromal cells of the bone. Stimulation of the normal osteoblasts and stromal cells by the tumor cells may then lead to secondary osteoclastogenesis, resulting in the osteolytic phenotype common to breast metastases.
\end{abstract}

\section{Citation of original article 2:}

R. B. Riggins, A. Zwart, R. Nehra, R. Clarke. The nuclear factor kappaB inhibitor parthenolide restores ICI 182,780 (Faslodex; fulvestrant)-induced apoptosis in antiestrogen-resistant breast cancer cells. Molecular Cancer Therapeutics 2005; 4(1): 33-41.

\begin{abstract}
of the original article 2
The molecular mechanisms underlying the acquisition of resistance to the antiestrogen Faslodex are poorly understood, although enhanced expression and activity of nuclear factor kappaB (NFkappaB) have been implicated as a critical element of this phenotype. The purpose of this study was to elucidate the mechanism by which NFkappaB up-regulation contributes to Faslodex resistance and to determine whether pharmacologic inhibition of NFkappaB by the small molecule parthenolide could restore Faslodex-mediated suppression of cell growth. Basal expression of multiple NFkappaB-related molecules in MCF7-derived LCC1 (antiestrogen-sensitive) and LCC9 (antiestrogen-resistant) breast cancer cells was determined, and cells were treated with Faslodex or parthenolide. The effect of these drugs either singly or in combination was assessed by cell proliferation, estrogen receptor (ER)-dependent transcriptional activation, cell cycle analysis, and apoptosis assays. Expression of the p65 NFkappaB subunit and the upstream NFkappaB regulator IkappaB kinase gamma/NFkappaB essential modulator were increased in the resistant MCF7/LCC9 cells $(P=0.001$ and 0.04 ,
\end{abstract}

Correspondence to: Christopher C. Benz, MD, Director, Cancer and Developmental Therapeutics Program, Buck Institute for Age Research, 8001 Redwood Boulevard, Novato, CA 94945, USA. E-mail: cbenz@buckinstitute.org; Tel: +1415 209 2092; Fax: +1 4152092232

Publication date 31/05/05

BCO/442/2005/JC 
respectively). Whereas MCF7/LCC9 cells were unresponsive to Faslodex alone, parthenolide effectively inhibited MCF7/LCC9 cell proliferation and the combination of Faslodex and parthenolide resulted in a 4-fold synergistic reduction in cell growth $(P=0.03)$. This corresponded to a restoration of Faslodex-induced apoptosis $(P=0.001)$, with no observable changes in ER-dependent transcription or cell cycle phase distribution.

Because parthenolide has shown safety in Phase I clinical trials, these findings have direct clinical relevance and provide support for the design of clinical studies combining antiestrogens and parthenolide in ER-positive breast cancer.

\section{Review}

The nuclear factor-кB (NFkB) complex encompasses a family of inducible transcription factors expressed in almost all cells and mediating a signaling pathway now recognized as lying 'at the crossroads of life and death' [1]. NFкB activation occurs in response to extracellular chemical stresses, cytokines, and growth factors; and directly regulates the expression of hundreds of genes whose cellular influences extend well beyond those of the immune system where its critical role was first recognized almost two decades ago [2]. The NFKB family consists of five mammalian members ( $p 50 / N_{\kappa} B 1$, p52/NFкB2, p65/relA, c-rel, and relB), all share a conserved 300 amino acid N-terminal Rel homology domain (homologous to that encoded by the avian oncogene, v-Rel) responsible for their dimerization, nuclear translocation, DNA-binding, and association with I $\mathrm{kB}$ inhibitory proteins [3,4]. All exist as homoor heterodimers (the most abundant form is generally thought to be the p50/p65 heterodimer), although in resting cells NFKB is cytoplasmically sequestered as a latent complex bound to one or more members of the I $\mathrm{k} B$ protein family $\left(\mathrm{I}_{\kappa} \mathrm{B} \alpha, \mathrm{I}_{\kappa} \mathrm{B} \beta, \mathrm{I}_{\kappa} \mathrm{B} \varepsilon, \mathrm{I}_{\kappa} \mathrm{B} \gamma\right.$, $\mathrm{Bcl}-3$, and the precursor Rel proteins p100 and p105). Through different signaling intermediates, various NFKB stimuli (e.g. TNF $\alpha$, CD40 ligand, IL-1, LPS, TRANCE/receptor activator of nuclear factor- $\kappa B$ ligand (RANKL), EGF, phorbol esters, peroxides, ionizing radiation) converge to activate $I_{\kappa} B$ kinase (IKK), a heterotrimeric cytoplasmic complex with two catalytic kinase subunits (IKK $\alpha$ and IKK $\beta$, either of which may be phosphorylated by upstream kinases) and a regulatory scaffolding component (IKK $\gamma / \mathrm{NEMO})$. This activated IKK complex in turn phosphorylates $\mathrm{I}_{\mathrm{K}} \mathrm{B}$, leading to its proteasomal degradation and translocation of NFKB into the nucleus, where it binds promoter-specific $\kappa B$ consensus DNA elements to direct transcription of over 180 known NFkB target genes.

$N F \kappa B$ regulated genes are known to affect a wide variety of anti-apoptotic, proliferative, motility and invasion promoting cellular responses critical for normal organ development and homeostasis, including that of the mammary gland [5]. Thus, it is not surprising that within the past decade we have seen a rapid surge in the number of reports implicating $\mathrm{NF}_{\kappa} \mathrm{B}$ dysregulation in various chronic inflammatory disorders and assorted hematopoietic and epithelial malignancies, and identifying this pathway as a promising target for new molecular therapies [6-10]. Among the experimental and medicinal strategies shown to inhibit constitutively active NFKB are drugs that target either upstream NFkB activating signals or downstream $І_{\kappa} B$ degradative mechanisms [11], including the potent and specific antioxidant pyrrolidine dithiocarbamate [12], proteasome inhibitors like MG-132 and PS-341 (bortezomib/velcade) [7], and sesquiterpene lactones like parthenolide (PA), a specific IKK inhibitor and component of feverfew now being evaluated in cancer patients $[13,14]$.

Two new breast cancer studies, Bhatia et al. [15] and Riggins et al. [16], implicate distinct but equally devious roles for $\mathrm{NF} K \mathrm{~B}$ dysregulation in the clinical progression of breast cancer. Both reports offer compelling findings that need further validation and mechanistic clarification. The immunohistochemical study of Bhatia et al. evaluated tumor expression of RANKL, which activates NFKB upon binding to the surface receptor, receptor activator of nuclear factorkappaB (RANK). They hypothesized that differential expression of RANKL or RANK might identify cancer cells predisposed to metastasis, and in particular formation of bony metastases associated with osteolytic complications. RANK is also known as osteoclast differentiation and activation receptor for its essential role in bone remodeling, mediated by osteoclastic activation of $\mathrm{NF} \mathrm{B}$, and bone resorption. RANKL, widely expressed on the surface of both osteoblasts and bone stroma, can bind to either cellular RANK or the soluble decoy receptor, osteoprotegerin (OPG), also known as osteoclastogenesis inhibitory factor because of its role in preserving bone density. For example, the bone mineral preserving activity of estrogen is mediated by stromal estrogen receptor (ER)-induction of OPG; and in an opposite manner, excess glucocorticoids can demineralize bone by inhibiting synthesis of OPG. Thus, local bone density and homeostasis are regulated by the competitive balance between OPG and RANK for binding to RANKL [17]. Comparing 
paraffin-embedded samples of nonneoplastic breast $(n=10$, NNB), infiltrating ductal carcinomas ( $n=58$, IDC; 26 nonmetastatic and 32 metastatic), and breast cancer bony metastases $(n=43$, breast cancer bony metastases (BTM)), Bhatia et al. observed no difference in the uniformly strong surface expression of RANK in all NNB, IDC, and BTM tissues analyzed. In contrast, they observed significantly decreased or absent surface expression of RANKL by BTM (only $2 \%$ positive) and IDC (31\% positive for metastatic IDC, $62 \%$ positive for nonmetastatic IDC), relative to NNB (90\% positive). Their findings that virtually all NNB samples express both RANK and RANKL are consistent with earlier observations that intact $\mathrm{NF} K \mathrm{~B}$ signaling is required for normal mammary organogenesis, that mice lacking RANK or RANKL fail to form lobuloalveolar structures during pregnancy, and that normal bone metabolism and mammary gland development are linked by NFKB responses to calcium demand [5]. Bhatia et al. speculate that decreased expression of RANKL by RANK-positive IDC allows for metastatic bone seeding by IDC and interaction with RANKLpositive stroma that induces osteolysis. While an attractive hypothesis, there is insufficient clinical information provided to confirm this proposal. Likewise, Bhatia et al. do not provide other relevant information, such as the ER status of their different study samples.

ER-positive IDC, while generally more indolent than ER-negative IDC, preferentially metastasizes to bone [18]; and this well recognized clinical fact raises an interesting issue not addressed by Bhatia et al. RANKL-negative IDC bony metastases would be expected to have activated NFKB (by interaction of their surface RANK with RANK-positive stroma), and this proliferation and survival signal would serve to augment the clinical invasiveness of these ER-positive metastatic cells. Until recently, however, ER-positive breast cancers were said to lack activated $N F_{\kappa} B$ on the basis of limited breast cancer surveys and the known inhibitory effects of activated NFKB on all steroid receptors including ER [19]. A more extensive breast cancer evaluation has now reported important new observations about $\mathrm{NF} K \mathrm{~B}$ activation in ER-positive primary breast cancers [20]: (i) those destined for later metastatic relapse express significantly higher levels of NFKB as compared to primary tumors that do not relapse; (ii) those with higher NFKB levels appear clinically resistant to adjuvant endocrine therapy by the antiestrogen tamoxifen; and (iii) the antiestrogen resistance associated with higher NFKB levels may be reversed by anti-NFKB agents like bortezomib or parthenolide. This newly recognized and potentially important clinical role of NFKB activation in determining the antiestrogen responsiveness of ER-positive breast cancers is the focus of the preclinical study by Riggins et al. [16], who evaluated NFkB expression in an ER-positive human breast cancer cell line model (MCF7/LCC9) resistant to the pure antiestrogen, fulvestrant (ICl-182,780).

Several laboratories have transfected ER-positive, tamoxifen-sensitive MCF-7 cells to show that constitutively activated receptor tyrosine kinases like ErbB2 and EGFR, or downstream kinases like Raf-1, MEK1 or Akt, all reduce ER levels and tamoxifen sensitivity; and importantly, ER levels and tamoxifen sensitivity can be restored in these endocrine-resistant sublines using $\mathrm{NF}_{\kappa} \mathrm{B}$ inhibiting doses of parthenolide [20-22]. In contrast to these studies, Riggins et al. employed two different MCF-7 sublines developed under long-term selection conditions: fulvestrant-resistant MCF7/LCC9 cells and fulvestrant-sensitive but estrogen-independent MCF7/LCC1 cells [16]. An earlier study by this same laboratory had shown that MCF7/LCC1 cells possessed increased NFKB activity relative to parental MCF-7 cells, and that the MCF7/LCC1 phenotype could be restored to an estrogen-dependent state by downregulation of NFKB [23]. Riggins et al. now report that fulvestrant-resistant MCF7/LCC9 cells exhibit significantly increased $\mathrm{NF} \kappa \mathrm{B}$ expression (and activating kinase, NEMO/IKK $\gamma$ ) relative to MCF7/LCC1 cells. Moreover, parthenolide can restore fulvestrant sensitivity to these MCF7/LCC9 cells and the combination parthenolide-fulvestrant produces synergistic inhibition of MCF7/LCC9 growth based on enhanced induction of apoptosis. While the restorative and antiestrogen potentiating effects of parthenolide are presumed due to parthenolide's IKK inhibitory activity as others have shown [20], Riggins et al. showed no change in intracellular $\mathrm{I}_{\kappa} \mathrm{B} \alpha$ levels and could not confirm under their experimental conditions that parthenolide actually inhibited NFKB activity. Thus, these investigators were forced to conclude that parthenolide might be interacting synergistically with fulvestrant in MCF7/LCC9 cells by 'other alternative mechanisms' [16].

Validating in vivo studies are clearly needed to support the general conclusions reported to date using these various NFKB-stimulated, ER-positive, and endocrine-resistant breast cancer cell line models [16,20-22]. Anticipating such in vivo validation, it is not too early to begin designing clinical trials in which antiestrogens like tamoxifen or fulvestrant are combined with anti-NFKB agents like parthenolide or bortezomib to treat patients with endocrine refractory ER-positive breast cancer. Future in vivo studies might also attempt to confirm another potential benefit predicted by the findings from Bhatia et al. [15], that anti-NFKB therapeutics can disrupt or prevent the devious interplay between bone and tumor cells that results in osteolytic metastases. 


\section{Acknowledgments}

Supported in part by National Institutes of Health sponsored grants R01-CA36773, R01-CA71468, R01-AG20521, and P50-CA58207.

\section{References}

1. Karin $\mathrm{M}$, Lin $\mathrm{A} . \mathrm{NF}-\kappa \mathrm{B}$ at the crossroads of life and death. Nat Immunol 2002; 3: 221-227.

2. Pahl HL. Activators and target genes of Rel/NF-kappaB transcription factors. Oncogene 1999; 18: 6853-6866.

3. Dixit V, Mak TW. NF-kappaB signaling: many roads lead to Madrid. Cell 2002; 111: 615-619.

4. Ghosh S, Karin M. Missing pieces in the NF-кB puzzle. Cell 2002; 109: 81s-96s.

5. Cao Y, Karin M. NF-kappaB in mammary gland: development and breast cancer. I Mammary Gland Biol Neoplasia 2003; 8: 215-223.

6. Baldwin AS. Control of oncogenesis and cancer therapy resistance by the transcription factor NF-кB. J Clin Invest 2001; 107: 241-246.

7. Feinman R, Siegel DS, Berenson J. Regulation of NF-кB in multiple myeloma: therapeutic implications. Clin Adv Hematol Oncol 2004; 2: 162-166.

8. Giardina C, Hubbard AK. Growing old with nuclear factorkappaB. Cell Stress Chaperon 2002; 7: 207-212.

9. Karin M, Yamamoto Y, Wang QM. The IKK NF-kappa B system: a treasure trove for drug development. Nat Rev Drug Discov 2004; 3: 17-26.

10. Wu JT, Kral JG. The NF-кB/lкB signaling system: a molecular target in breast cancer therapy. $J$ Surg Res 2005; 123: 158-169.

11. Yamamoto Y, Gaynor RB. Therapeutic potential of inhibition of the NF-kappa B pathway in the treatment of inflammation and cancer. J Clin Invest 2001; 107: 135-142.

12. Schreck R, Meier B, Mannel DN, et al. Dithiocarbamates as potent inhibitors of nuclear factor kappa B activation in intact cells. J Exp Med 1992; 175: 1181-1194.

13. Hehner SP, Hofmann TG, Droge W, Schmitz ML. The anti-inflammatory sesquiterpene lactone parthenolide inhibits NF-kappaB by targeting the IkappaB kinase complex. J Immunol 1999; 163: 5617-5623.

14. Curry III EA, Murry DJ, Yoder C, et al. Phase I dose escalation trial of feverfew with standardized doses of parthenolide in patients with cancer. Invest New Drug 2004; 22: 299-305.

15. Bhatia P, Sander MM, Hansen MF. Expression of receptor activator of nuclear factor- $\mathrm{B}$ is inversely correlated with metastatic phenotype in breast carcinoma. Clin Cancer Res 2005; 11: 162-165.

16. Riggins RB, Zwart A, Nehra R, Clarke R. The nuclear factor kB inhibitor parthenolide restores ICl 182,780 (faslodex; fulvestrant)-induced apoptosis in antiestrogenresistant breast cancer cells. Mol Cancer Ther 2005; 4: 33-41.

17. Walsh MC, Choi Y. Biology of the TRANCE axis. Cytokine Growth Factor Rev 2003; 14: 251-263.

18. Hess KR, Pusztai L, Buzdar AU, Hortobagyi GN. Estrogen receptors and distinct patterns of breast cancer relapse. Breast Cancer Res Treat 2003; 78: 105-118.

19. Nakshatri $H$, Bhat-Nakshatri $P$, Martin DA, et al. Constitutive activation of NF-kappaB during progression of breast cancer to hormone-independent growth. Mol Cell Biol 1997; 17: 3629-3639.

20. Zhou Y, Eppenberger-Castori S, Marx C, et al. Activation

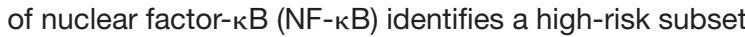
of hormone-dependent breast cancers. Int $\mathrm{J}$ Biochem Cell Biol 2005; 37: 1130-1144.

21. deGraffenried LA, Chandrasekar B, Friedrichs WE, et al. NF- $\mathrm{B}$ inhibition markedly enhances sensitivity of resistant breast tumor cells to tamoxifen. Ann Oncol 2004; 15: 885-890.

22. Holloway JN, Murthy S, El-Ashry D. A cytoplasmic substrate of mitogen-activated protein kinase is responsible for estrogen receptor- $\alpha$ down-regulation in breast cancer cells: the role of nuclear factor-кB. Mol Endocrinol 2004; 18: $1396-1410$

23. Pratt MA, Bishop TE, White D, et al. Estrogen withdrawal-induced NF-kappaB activity and bcl-3 expression in breast cancer cells: roles in growth and hormone independence. Mol Cell Biol 2003; 23: 6887-6900. 